\title{
Effects of Spaceflight on Human Skin
}

\author{
Árpád Farkas ${ }^{\mathrm{a}}$ Gergő Farkas ${ }^{\mathrm{b}}$

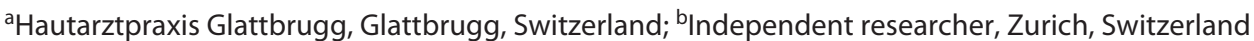

\section{Keywords}

Spaceflight · International Space Station · Microgravity ·

Astronaut · Skin $\cdot$ Immune system $\cdot$ Microbiome

\begin{abstract}
During both short- and long-duration spaceflight, several health problems can occur, including those of the skin. Astronauts in space and after returning to earth experience erythematous, burning, itchy, dry, sensitive, and thinning skin. Other skin problems, such as infections, abrasions, lacerations, delayed wound healing, and accelerated skin aging, are also common. Human skin is an ecosystem composed of a wide range of habitats for bacteria, fungi, and viruses called microbiome, which not only show a strong skin site-specific preference but also serve as microbial fingerprints that are highly unique to individuals. These human skin-associated microorganisms make a substantial contribution to the microbial ecosystems that inhabit the closed environments in space. On the other hand, human skin microbiome is also subject to change during spaceflight, which may lead to skin infections or the flare up of skin diseases. This review highlights some of the interactions between the space environment and the skin.

(c) 2021 S. Karger AG, Basel
\end{abstract}

karger@karger.com

(c) 2021 S. Karger AG, Basel

www.karger.com/spp

Karger!

\section{Introduction}

The International Space Station (ISS) is a large closed habitable construct located approximately $400 \mathrm{~km}$ above the earth. Various life support systems sustain conditions for humans to work productively and safely. Air pressure, temperature, humidity, and contamination must be kept under control [1]. The evaluation of the future of both noncommercial and commercial human spaceflight in low-Earth orbit, including long-range opportunities for the ISS and other free-flying structures, is ongoing. Human missions are planned to return to the surface of the Moon and to land on Mars. Microgravity environment, high radiation levels, continuous work and stress aboard the ISS, other free-flying structures, and spacecrafts are the main factors affecting human health over a time span of minutes to months. Spaceflight alters the cardiovascular, musculoskeletal, respiratory, vestibular, visual, and immune systems [2]. Simultaneously, astronauts face neurocognitive and neuropsychological changes [3]. Water supplies on the ISS are very limited, and the methods that astronauts use to maintain good hygiene in microgravity are not the same as on earth. Astronauts, for example, are unable to take a bath or a shower; therefore, they keep their body clean by using wet tissues and they 
Table 1. In-flight skin-related clinical symptoms and medical conditions which may occur, flare up, or worsen on board the ISS

\begin{tabular}{ll}
\hline Clinical symptoms & Medical conditions \\
\hline Erythema & Psoriasis \\
Burning & Seborrheic dermatitis \\
Pruritus & Atopic dermatitis \\
Peeling & Contact dermatitis \\
Dryness & Folliculitis \\
Sensitivity & Rosacea \\
Irritation & Acne \\
Thinning & Bacterial infections \\
Abrasions & Viral infections \\
Bruising & Fungal infections \\
Fluid shift & Hypersensitivities \\
Ecchymosis & Allergies \\
& Frostbite \\
& Delayed wound healing \\
& Onycholysis \\
\hline
\end{tabular}

ISS, International Space Station.

wash their hair with a rinseless shampoo. They do not have a washing machine and they cannot change their clothes as often as on earth. These and other circumstances, such as temperature change, microgravity, radiation, and direct impairing factors, very much affect their skin health and lead to frequent skin impairments and skin diseases. The human skin is host to a complex and rich microbial community, called microbiome. Spaceflight is associated with strong and specific microbiome alterations. Understanding the nature of skin alterations and of the microbiome is key to managing astronaut health and maintenance of the ISS, other free-flying structures, and spacecraft equipment. In this article, the main factors encountered in space and their impact on human skin will be reviewed.

\section{Human Skin Impairments and Diseases during Space Missions}

Based on the medical records it seems that cutaneous alterations represent a major concern for astronauts. Erythema, peeling, dryness, burning, pruritus, sensitivity, thinning, and delayed wound healing are frequent skin problems [4-11]. Space suit, glove, boot, celestial dust, and lunar soil can cause significant trauma to crew members, such as irritation, bruising, and abrasion, and gloveinduced frostbite, ecchymosis, and onycholysis were re- corded [12-16]. Skin diseases such as psoriasis, seborrheic dermatitis, atopic dermatitis, contact dermatitis, folliculitis, rosacea, and acne may flare up $[4,8,10,17$, $18]$. Bacterial, fungal, and viral infections $[9,19-22]$ may occur and reactivation of herpesviruses causing herpes zoster was noted $[4,23]$ and increased incidence of hypersensitivities and of allergies were documented [9] (Table 1).

The National Aeronautics and Space Administration (NASA) created the Longitudinal Study of Astronaut Health in 1992 to address a variety of issues, including both the health of astronauts during spaceflight and the longer term health issues that might be associated with spaceflight and flight training including the possibility that increased exposure to radiation in space might have an impact on skin cancer cases. The study found 33 cases of basal and squamous cell carcinomas of the skin among 312 astronauts compared to 27 cases among 912 controls, an almost 3 -fold difference in rate, which is statistically significant. All these cases of basal and squamous cell carcinomas of the skin were excluded from the analysis of the astronaut group and of the comparison group. The reason for this deletion is that astronauts spend significant time outdoors for both training and recreational purposes; therefore, these results have to be interpreted carefully [24].

\section{Skin Physiology in Microgravity: Dermal Atrophy, Cutaneous Muscle, Hair Follicle, and Metabolic Alterations}

During the SkinCare experiment, skin surface, epidermis, and dermis were analyzed preflight, in-flight, and postflight of an ISS astronaut. One measuring field of the 2 inner forearms was treated with a skincare emulsion. It seemed that after a prolonged stay in weightlessness, the skin underwent an accelerated aging with a thinner, more structured, coarser epidermis and a loss of skin elasticity. These changes appeared to be reversible because after a year the skin's condition returned to normal [5].

Later the Skin-B project was launched to validate the results of the SkinCare study on 6 astronauts. In orbit, skin hydration, skin barrier function, and surface evaluation of the living skin (SELS; VisioScan ${ }^{\circledR}$ VC98 camera) were measured. On the ground, skin elasticity, skin density, skin thickness, and skin microcirculation were evaluated. An improvement was seen in skin hydration and skin barrier function. No changes or improvement was observed in the appearance of the skin surface. Skin density, skin thickness, and skin elasticity values were un- 
changed from preflight values. These findings correspond to some reports from other astronauts after returning from flight. Quicker skin healing, decreased skin cracking over the course of the mission, and use of a lower amount of skin lotion than on earth was documented. The authors of the Skin-B investigation explain these differences by improved space conditions such as the use of better exercise devices and better nutrition on board the ISS [25]. During the Skin-B project, the authors measured the skin surface profile by means of SELS and after interpreting their results they concluded that it is necessary to take a closer look at the fluid shift phenomenon when an increased volume of fluid travels toward the upper regions of the body, making them look swollen. They found that only the parameter skin volume leads to meaningful data under microgravity and the formulas for the SELS parameters, such as roughness, scaling, and wrinkles, need to be reevaluated [26]. These observations highlight the importance of carefully selecting and adapting the experimental methods during microgravity studies.

A rodent study which involved 3 mice having spent 3 months in orbit showed a significant reduction in dermal thickness accompanied by increased newly synthetized procollagen. Transcriptomic data suggested that the dermal atrophy might be related to an early degradation of defective newly formed procollagen molecules. Many hair follicles in the growing anagen phase were observed, validated by an increased expression of specific hair follicle genes. Additionally the analysis of whole thickness skin showed that a large proportion of the upregulated transcripts encoded proteins related to striated muscle homeostasis [27].

In summary, data from the Skin-B study contradicted the SkinCare results. During the SkinCare experiment, a reduction in skin density, a loss of skin elasticity, and reduced skin thickness were observed. These skin physiology parameters remained unchanged during the Skin-B study and skin hydration and skin barrier function even changed for the better. The above mentioned rodent study confirmed the reduced skin thickness in agreement with the data obtained in the SkinCare experiment. However, these changes should be the subject of further investigations.

Another study which analyzed the hair follicles of 10 astronauts showed that genes related to hair growth, such as Homo sapiens fibroblast growth factor 18, Homo sapiens angiopoietin-like 7, and Homo sapiens cartilage oligomeric matrix protein, were upregulated during flight, suggesting that spaceflight inhibits cell proliferation in hair follicles [28].

Skin in Spaceflight
A mouse study during a Space Shuttle Atlantis (STS135) mission showed that in the flight group many genes responsible for regulating production and metabolism of reactive oxygen species were significantly altered with 1.5 -fold changes compared to controls. For extracellular matrix profile, several genes encoding matrix and metalloproteinases involved in extracellular matrix remodeling were significantly up-/downregulated. Of 332 biochemical markers, 19 differed significantly, with 12 upregulated and 7 downregulated including altered amino acid, carbohydrate metabolism, cell signaling, and transmethylation pathways. The data demonstrated that spaceflight condition leads to a shift in biological and metabolic homeostasis as a consequence of increased regulation in cellular antioxidants, reactive oxygen species production, and tissue remodeling [29].

\section{Environmental Biocontamination on Board the ISS and Skin Microbiome}

Diverse bacteria, fungi, and viruses inhabit the human skin. These microorganisms have an individual pattern and vary between different skin sites. Some microbes join the ISS when new crew members or payload arrives and some were inhabitants from the very beginning when the station was assembled.

The initial colonization of surfaces on board the Russian segment of the ISS was dominated by Gram-positive members of the genera Staphylococcus, Micrococcus, $\mathrm{Ba}$ cillus, and Streptococcus showing that the skin of crew members was the primary source of early contamination. Gram-negative bacteria and fungi were also present [30]. Predominant bacteria belonged to the members of the Staphylococcus genus, isolated from $84 \%$ of air and surface samples $[31,32]$. In air samples, Aspergillus and Penicillium were the dominant fungi, while on the surface samples, Aspergillus and Cladosporium dominated [32, 33]. Similar observations have been made after sampling of NASA's Destiny Laboratory $[34,35]$ and Japan Aerospace Exploration Agency's Kibo facility aboard the ISS, where earlier Alternaria and Malassezia species were also detected [35-37].

Staphylococcus species isolated from ISS air and crew members were resistant to at least 1 antibiotic used aboard the ISS and most were capable of surface colonization and subsequent biofilm formation [38]. Rifampicin resistance due to higher mutation frequencies of Staphylococcus epidermidis cultivated in the ISS compared to ground controls was confirmed during an investigation as part of the

Skin Pharmacol Physiol 2021;34:239-245 
Table 2. Skin-related microbial species which play a role on board the ISS

\begin{tabular}{ll}
\hline Bacterial species & Fungal species \\
\hline Staphylococcus & Malassezia \\
Corynebacterium & Cladosporium \\
Micrococcus & Cyberlindnera \\
Propionibacterium & Candida \\
Streptococcus & Trichosporon \\
Sporosarcina & Cryptococcus \\
Actinomyces & Penicillium \\
Rothia & Saccharomyces \\
Haemophilus & Aspergillus \\
Pseudomonas & Alternaria \\
Finegoldia & \\
\hline
\end{tabular}

ISS, International Space Station.

SpaceX-3 resupply mission showing that the human spaceflight environment leads to changes in bacterial mutagenic potential [39]. Many other studies were performed, which analyzed ISS surface and air samples to assess the succession and persistence of microbial communities, their antimicrobial resistance profiles, and virulence properties. These investigations revealed the richness of microbial diversity inside the ISS and confirmed previous results showing that skin-associated bacterial and fungal species play an important role. Some of them belong to risk group microorganisms showing antimicrobial resistance signatures associated with $\beta$-lactam, cationic antimicrobial peptide, and vancomycin. Prominent virulence factors were cobalt-zinc-cadmium resistance and multidrug resistance efflux pumps [40-50]. Table 2 summarizes the most important skin-related microbial species, which play a role on board the ISS.

One study has investigated the microbiome changes of 9 astronauts who spent $6-12$ months in the ISS [51]. The ISS environment differentially influenced the forehead and forearm skin microbiota. Alpha diversity and richness increased or decreased, depending upon the individual, but was consistent between the forehead and forearm skin. In spite of the bimodal variance amongst astronaut skin microbiomes, there was a common shift in the microbial composition of all crew members. These changes involved a significant in-flight reduction of Proteobacteria, mostly Gammaproteobacteria and Betaproteobacteria, with a concomitant increase in Firmicutes, including staphylococcal and streptococcal species. Different factors may contribute to the decreased amount of Gammaproteobacteria and Betaproteobacteria abundance such as the lack of a "green" natural environment plus the constant filtration of air and the alteration of the skin structure during spaceflight. The reduction of skin Gammaproteobacteria might then contribute to the high frequency of skin hypersensitivity reactions/rashes and skin infections. The change in skin structure might also facilitate the establishment of skin infections by opportunistic pathogens such as the staphylococcal and streptococcal species [51].

\section{Discussion}

Human spaceflight activities in low-Earth orbit are in transition as governments in the future become only one of the possible customers for commercial services. There is an emerging industry that is close to becoming a reality: space tourism. In the near future, lunar surface operations and missions beyond cislunar space will help the return of astronauts to the surface of the Moon and capabilities are being developed for human missions to Mars and other destinations.

Therefore, it is getting more important to better understand human health issues during these missions and optimize healthcare in space, which includes optimal body cleaning methods and the use of moisturizers, and to address improper space suit, glove, and boot fit-induced skin breakdown $[15,16]$. In microgravity, desiccated skin particles or foot calluses simply float; therefore, the station's ventilation system should work properly.

The use of the NASA imaging systems facilitates the diagnosis of health-related conditions and the crew's surgeons periodically discuss all health issues of each crew member to initiate a treatment with proper medication. Telemedicine may function well for the astronauts on the ISS, but as they get further away from Earth they will have an increased communication delay so the approach to medical care will need to change. Crews need to be more autonomous and there needs to be a physician on board.

Skin cancer prevention studies have to be implemented and the relationship between radiation and microgravity has to be further investigated. Common stick insect (Carausius morosus) experiments showed abnormal differentiation under space microgravity and recessive lethal mutations were induced by space radiation in the progeny of fruit flies showing that microgravity and space radiation may together elevate mutation frequencies. It was suggested that DNA damage repair inhibition and stressrelated protein accumulation were involved. On the contrary, some space experiments have shown that mutation 
frequencies and DNA repair activity were not affected by microgravity in Escherichia coli, Bacillus subtilis, Dictyostelium discoideum, Saccharomyces cerevisiae, and human cells. Measurements of space radiation in the Shuttle-Mir mission 9 showed that doses differed between tissues and organs of the human body with effective doses varying by $10 \%$ or more. There was a tendency to have a high dose on the surface of the body (skin) and protruding near surface elements (e.g., bone surfaces corresponding to the shoulder) and to have low doses in internal regions (e.g., bone marrow and colon). These data highlight the importance of radiation protection systems for travel in space [52].

The dysregulation of the human immune system in space includes altered leukocyte, monocyte, and granulocyte function, altered cytokine production patterns, abrogated bone marrow responsiveness to colony-stimulating factors, altered T-cell intracellular signaling, persistent hypersensitivity reactions, inhibition of natural killer cell activity, and apparent $\mathrm{T}$ helper 2 potential bias shift. This means that astronauts are more susceptible to infections. Interventions for reducing the risk of infections prior to space journeys include vaccinations. Because of the possible reactivation of herpesviruses, varicella zoster virus vaccine should be given to mention one. Screening of astronauts for both methicillin-susceptible and methicillinresistant Staphylococcus aureus is worth considering and Staphylococcus aureus carriers may be decolonized. Recent research has demonstrated that colonizing the skin and nares with extracellular serine protease-producing Staphylococcus epidermidis selectively inhibits Staphylococcus aureus nasal colonization and biofilm formation. As such, a somewhat far reaching countermeasure could involve colonizing astronauts' skin and nares prior to travel with extracellular serine protease-producing Staphylococcus epidermidis $[53,54]$.

To mimic spaceflight conditions ground-based habitats have been built such as the Antarctic Concordia Station and isolation facilities like an inflated lunar/Mars analog habitat, the Hawai'i Space Exploration Analog and Simulation isolation habitat, and the Mars500 facility. The Mars500 project simulated a crewed 520-day-long return flight to Mars. Cultivation assays revealed a Staphylococcus- and Bacillus-dominated microbial community on various surfaces, with a microbial load that did not exceed the allowed limits for ISS in-flight requirements. Mainly the crew was responsible for microbial dispersal. Opportunistic pathogens, stress-tolerant or potentially mobile element-bearing microorganisms were prevalent, while the overall microbial diversity dropped significant- ly over time [55]. It is therefore important for the advancement of spaceflight activities to properly govern microbial communities. Data from these ground-based facilities may help to estimate future risk of crewed spaceflight, could facilitate the design of a more optimal spacecraft mission, and can promote to choose appropriate microbial monitoring methods and potential countermeasures to ensure a microbiologically safe spaceflight environment.

A recent NASA study, which compared the impact of the spaceflight environment on a pair of monozygotic (identical) twin astronauts, highlights the importance of the measurement of the same variables in an astronaut and in his Earth-bound twin, because previous studies could not integrate simultaneous effects on multiple systems and data types in the same subject. One of the previous observations confirmed by this study was that some physiological adaptations, such as headward fluid shift, are rapid and reach a new steady state within days [56]. However, this fluid shift has an impact during measurements on the parameter volume, skin roughness, skin scaling, and skin wrinkles. This has to be taken into consideration when methods such as SELS are being used [25].

Further and ongoing tests on more subjects are needed to minimize space-induced skin impairments. The possible lessons learned with space exploration will also have an impact on human health on Earth. In fact, this is a productive, cross-fertilized, endeavor in which studies performed on Earth yield countermeasures for protection of space crew health and space research is translated into health measures for the Earth-bound population.

\section{Conflict of Interest Statement}

The authors have no conflicts of interest to declare.

\section{Funding Sources}

The authors did not receive any funding.

\section{Author Contributions}

Árpád Farkas performed a comprehensive literature search in the databases, contacted experts, and wrote the article. Gergö Farkas suggested the structure of the article and corrected the article. 


\section{Data Availability Statement}

The authors provided a systematic critical digest of English articles using MEDLINE search (2000-2020). Additional information, articles, publications, and abstracts were identified by reading previously published reviews, by searching NASA databases and using ResearchGate (1974-2020). Experts in the field were also contacted. Search terms included space, spaceflight, astronaut, microgravity, international space station, ISS, skin, dermatology, microbiome, immune system, telemedicine, and teledermatology.

\section{References}

1 Gentry GJ, Cover J. International space station (ISS) environmental control and life support (ECLS) system overview of events: 20102014. 45th International Conference on Environmental Systems; 2015. p. 1-12 [Online]. Available from: https://ntrs.nasa.gov/api/citations/20150022318/downloads/ 20150022318.pdf. Accessed 2020 Dec 8.

2 Demontis GC, Germani MM, Caiani EG, Barravecchia I, Passino C, Angeloni D. Human pathophysiological adaptations to the space environment. Front Physiol. 2017;8:547.

3 De la Torre GG. Cognitive neuroscience in space. Life. 2014;4(3):281-94.

4 Wotring VE. Recent pharmacology studies on the International space station. 2014 [Online]. Available from: https://ntrs.nasa.gov/ api/citations/20140012420/downloads/20140012420.pdf. Accessed 2020 Dec 8.

5 Tronnier $\mathrm{H}$, Wiebusch M, Heinrich U. Change in skin physiological parameters in space: report on and results of the first study on man. Skin Pharmacol Physiol. 2008;21(5): 283-92.

6 Gontcharov IB, Kovachevich IV, Pool SL, Navinkov OL, Barratt MR, Bogomolov VV, et al. In-flight medical incidents in the NASAMir program. Aviat Space Environ Med. 2005;76(7):692-6.

7 Wotring VE. Medication use by U.S. Crewmembers on the International space station. FASEB J. 2015;29(11):4417-23.

8 Crucian B, Johnston S, Mehta S, Stowe R, Uchakin $\mathrm{P}$, Quiriarte $\mathrm{H}$, et al. A case of persistent skin rash and rhinitis with immune system dysregulation onboard the International space station. J Allergy Clin Immunol Pract. 2016;4(4):759-62.e8.

9 Crucian B, Babiak-Vazquez A, Johnston S, Pierson DL, Ott CM, Sams C. Incidence of clinical symptoms during long-duration orbital spaceflight. Int J Gen Med. 2016;9:38391.

10 Braun N, Thomas S, Tronnier H, Heinrich U. Self-reported skin changes by a selected number of astronauts after long-duration mission on iss as part of the skin B project. Skin Pharmacol Physiol. 2019;32(1):52-7.

11 Lowrey CR, Perry SD, Strzalkowski ND, Williams DR, Wood SJ, Bent LR. Selective skin sensitivity changes and sensory reweighting following short-duration space flight. J Appl Physiol. 2014;116(6):683-92.

12 Antonsen E, Bayuse T, Blue R, Daniels V, Hailey M, Hussey S, et al. Evidence report: risk of adverse health outcomes and decrements in performance due to in-flight medical conditions.
2018:1-80 [Online]. Available from: https://humanresearchroadmap.nasa.gov/evidence/reports/Medical.pdf. Accessed 2020 Dec 8.

13 Scheuring RA, Mathers CH, Jones JA, Wear ML. Musculoskeletal injuries and minor trauma in space: incidence and injury mechanisms in US astronauts. Aviat Sp Environ Med. 2009;80:117-24.

14 Scheuring R. Sports injuries and space injuries: prevention and treatment comparable populations? 2018 [Online]. Available from: https://ntrs.nasa.gov/api/citations/ $20160002368 /$ downloads/20160002368. pdf. Accessed 2020 Dec 8.

15 Charvat CM, Norcross J, Reid CR, McFarland SM. Spacesuit Glove-induced hand trauma and analysis of potentially related risk variables. 45th International Conference on Environmental Systems; 2015. p. 1-44 [Online]. Available from: https://ntrs.nasa.gov/citations/20150003049. Accessed 2020 Dec 8.

16 Diaz A, Anderson A, Kracik M, Trotti G, Hoffman J, Newman D. Development of a comprehensive astronaut spacesuit injury database. 63rd International Astronautical Congress. Naples, Italy [Online]. Available from: https://www.researchgate.net/publication/279200129_Development_of_a_Comprehensive_Astronaut_Spacesuit_Injury_ Database. Accessed 2020 Dec 8.

17 Hawkins W, Zieglschmid J. SP-368 Biomedical results of apollo. Section II, chapter 1: clinical aspects of crew health [Online]. Available from: http://history.nasa.gov/SP-368/s2ch1. htm. Accessed 2020 Dec 8.

18 Burgdorf WH, Hoenig LJ. Dermatology and the American experience in space. JAMA Dermatol. 2015;151(8):877.

19 Crucian B, Sams CF. Evidence report : risk of crew adverse health event due to altered immune response human health countermeasures element. 2015 [Online]. Available from: https://ntrs.nasa.gov/api/citations/ $20140002769 /$ downloads/20140002769. pdf. Accessed 2020 Dec 8.

20 Taylor GR. Recovery of medically important microorganisms from Apollo astronauts. Aerosp Med. 1974;45(8):824-8.

21 Mehta SK, Laudenslager ML, Stowe RP, Crucian BE, Sams CF, Pierson DL. Multiple latent viruses reactivate in astronauts during Space Shuttle missions. Brain Behav Immun. 2014;41:210-7.

22 Rooney BV, Crucian BE, Pierson DL, Laudenslager ML, Mehta SK. Herpes virus reactivation in astronauts during spaceflight and its application on earth. Front Microbiol. 2019;10:16-9.
23 Cohrs RJ, Mehta SK, Schmid DS, Gilden $\mathrm{DH}$, Pierson DL. Asymptomatic reactivation and shed of infectious varicella zoster virus in astronauts. J Med Virol. 2008;80(6): 1116-22.

24 Longnecker DE, Manning FJ, Worth MH, Institute of Medicine (US) Committee on the Longitudinal Study of Astronaut Health. Review of NASA's Longitudinal Study of astronaut health. Washington: National Academies Press; 2004.

25 Braun N, Binder S, Grosch H, Theek C, Ülker J, Tronnier H, et al. Current data on effects of long-term missions on the international space station on skin physiological parameters. Skin Pharmacol Physiol. 2019;32(1):43-51.

26 Theek C, Tronnier H, Heinrich U, Braun N. Surface evaluation of living skin (SELS) parameter correlation analysis using data taken from astronauts working under extreme conditions of microgravity. Skin Res Technol. 2020;26(1):105-11.

27 Neutelings T, Nusgens BV, Liu Y, Tavella S, Ruggiu A, Cancedda R, et al. Skin physiology in microgravity: a 3-month stay aboard ISS induces dermal atrophy and affects cutaneous muscle and hair follicles cycling in mice. NPJ Microgravity. 2015;1:15002.

28 Terada M, Seki M, Takahashi R, Yamada S, Higashibata A, Majima HJ, et al. Effects of a closed space environment on gene expression in hair follicles of astronauts in the International space station. PLoS One. 2016; 11:1-17.

29 Mao XW, Pecaut MJ, Stodieck LS, Ferguson VL, Bateman TA, Bouxsein ML, et al. Biological and metabolic response in STS-135 spaceflown mouse skin. Free Radic Res. 2014;48(8): $890-7$.

30 Reidt U, Helwig A, Plobner L, Lugmayr V, Treutlein U, Kharin S, et al. Study of initial colonization by environmental microorganisms in the russian segment of the international space station (ISS). Gravit Sp Res. 2014; 2:46-57.

31 Novikova ND. Review of the knowledge of microbial contamination of the Russian manned spacecraft. Microb Ecol. 2004;47(2): $127-32$.

32 Novikova N, De Boever P, Poddubko S, Deshevaya E, Polikarpov N, Rakova N, et al. Survey of environmental biocontamination on board the International space station. Res Microbiol. 2006;157(1):5-12.

33 Taylor PW. Impact of space flight on bacterial virulence and antibiotic susceptibility. Infect Drug Resist. 2015;8:249-62. 
34 Castro VA, Thrasher AN, Healy M, Ott CM, Pierson DL. Microbial characterization during the early habitation of the international space station. Microb Ecol. 2004;47(2):11926.

35 Yamaguchi N, Roberts M, Castro S, Oubre C, Makimura K, Leys N, et al. Microbial monitoring of crewed habitats in space-current status and future perspectives. Microbes Environ. 2014;29(3):250-60.

36 Satoh K, Nishiyama Y, Yamazaki T, Sugita T, Tsukii Y, Takatori K, et al. Microbe-I: fungal biota analyses of the Japanese experimental module KIBO of the International space station before launch and after being in orbit for about 460 days. Microbiol Immunol. 2011; 55(12):823-9.

37 Ichijo T, Hieda H, Ishihara R, Yamaguchi N, Nasu M. Bacterial monitoring with adhesive sheet in the International space station"Kibo", the Japanese experiment module. Microbes Environ. 2013;28(2):264-8.

38 Schiwon K, Arends K, Rogowski KM, Fürch S, Prescha K, Sakinc T, et al. Comparison of antibiotic resistance, biofilm formation and conjugative transfer of staphylococcus and enterococcus isolates from international space station and antarctic research station concordia. Microb Ecol. 2013;65(3):638-51.

39 Fajardo-Cavazos P, Nicholson WL. Cultivation of Staphylococcus epidermidis in the human spaceflight environment leads to alterations in the frequency and spectrum of spontaneous rifampicin-resistance mutations in the rpoB gene. Front Microbiol. 2016;7:99910.

40 Venkateswaran K, Vaishampayan P, Cisneros J, Pierson DL, Rogers SO, Perry J. International space station environmental microbiome: microbial inventories of ISS filter debris. Appl Microbiol Biotechnol. 2014;98(14): 6453-66.
41 Checinska A, Probst AJ, Vaishampayan P, White JR, Kumar D, Stepanov VG, et al. Microbiomes of the dust particles collected from the International space station and spacecraft assembly facilities. Microbiome. 2015;3:50.

42 Sugita T, Yamazaki T, Makimura K, Cho O, Yamada S, Ohshima $\mathrm{H}$, et al. Comprehensive analysis of the skin fungal microbiota of astronauts during a half-year stay at the International space station. Med Mycol. 2016;54(3):232-9.

43 Mora M, Perras A, Alekhova TA, Wink L, Krause R, Aleksandrova A, et al. Resilient microorganisms in dust samples of the International Space Station-survival of the adaptation specialists. Microbiome. 2016;4(1):65.

44 Be NA, Avila-Herrera A, Allen JE, Singh N, Checinska Sielaff A, Jaing C, et al. Whole metagenome profiles of particulates collected from the International space station. Microbiome. 2017;5(1):81.

45 Lang JM, Coil DA, Neches RY, Brown WE, Cavalier D, Severance M, et al. A microbial survey of the International space station (ISS). PeerJ. 2017;5:e4029-20.2017

46 Singh NK, Wood JM, Karouia F, Venkateswaran K. Succession and persistence of microbial communities and antimicrobial resistance genes associated with International Space Station environmental surfaces. Microbiome. 2018;6(1):1-23.

47 Singh NK, Wood JM, Karouia F, Venkateswaran K. Correction to: succession and persistence of microbial communities and antimicrobial resistance genes associated with International Space Station environmental surfaces. Microbiome. 2018;6.

48 Checinska Sielaff A, Mohan G, Karouia F, Minich J, Tran Q, Urbaniak C, et al. Characterization of the total and viable bacterial and fungal communities associated with the International Space Station surfaces. Microbiome. 2019;7:50.
49 Vaishampayan A, Grohmann E. Multi-resistant biofilm-forming pathogens on the International space station. J Biosci. 2019;44(5): $1-5$.

50 Avila-Herrera A, Thissen J, Urbaniak C, Be NA, Smith DJ, Karouia F, et al. Crewmember microbiome may influence microbial composition of ISS habitable surfaces. PLoS One. 2020;15:1-20.

51 Voorhies AA, Mark Ott C, Mehta S, Pierson DL, Crucian BE, Feiveson A, et al. Study of the impact of long-duration space missions at the International Space Station on the astronaut microbiome. Sci Rep. 2019;9(1):9911-7.

52 Ohnishi K, Ohnishi T. The biological effects of space radiation during long stays in space. Biol Sci Space. 2004;18(4):201-5.

53 Weinstein R, Mermel LA. Infection prevention and control during prolonged human space travel. Clin Infect Dis. 2013;56(1):12330.

54 Crucian BE, Choukèr A, Simpson RJ, Mehta S, Marshall G, Smith SM, et al. Immune system dysregulation during spaceflight: potential countermeasures for deep space exploration missions. Front Immunol. 2018;9:1-21.

55 Schwendner P, Mahnert A, Koskinen K Moissl-Eichinger C, Barczyk S, Wirth R, et al. Preparing for the crewed Mars journey: microbiota dynamics in the confined Mars500 habitat during simulated Mars flight and landing. Microbiome. 2017;5(1):129.

56 Garrett-Bakelman FE, Darshi M, Green SJ, Gur RC, Lin L, Macias BR, et al. The NASA twins study: a multidimensional analysis of a year-long human spaceflight. Science. 2019; 12(6436):364. 